\title{
REVISTA
}

Revista Educación

ISSN: 0379-7082

ISSN: 2215-2644

revedu@gmail.com

Universidad de Costa Rica

Costa Rica

\section{La coherencia curricular en la Educación Superior: algunas reflexiones}

\author{
González-García, Victoria \\ La coherencia curricular en la Educación Superior: algunas reflexiones \\ Revista Educación, vol. 42, núm. 2, 2018 \\ Universidad de Costa Rica, Costa Rica \\ Disponible en: http://www.redalyc.org/articulo.oa? id $=44055139040$ \\ DOI: https://doi.org/10.15517/revedu.v42i2.28515
}

Esta obra está bajo una Licencia Creative Commons Atribución-NoComercial-SinDerivar 3.0 Internacional. 


\section{La coherencia curricular en la Educación Superior: algunas reflexiones}

\section{Curriculum Coherence in Higher Education: Some Reflections}

Victoria González-García 1

Universidad de Costa Rica, Costa Rica

victoria.gonzalez@ucr.ac.cr

(D) http://orcid.org/0000-0001-7391-0142
DOI: https://doi.org/10.15517/revedu.v42i2.28515

Redalyc: http://www.redalyc.org/articulo.oa?id=44055139040

Recepción: 14 Abril 2017

Aprobación: 16 Octubre 2018

\section{Resumen:}

En el marco de la investigación Relacionando pedagogía, currículo y didáctica: el caso de las carreras de la Universidad de Costa Rica, reacreditadas ante el SINAES, en este ensayo se comparten algunas reflexiones teóricas desde una perspectiva hispanoamericana, enriquecidas con los aportes de personas entrevistadas, quienes coordinan o dirigen proyectos y programas vinculados directamente con la población docente de la Universidad de Costa Rica con el propósito de promover la mejora del desempeño docente. Es así que este trabajo se estructura en dos momentos. El primero, es una reflexión sobre educación, pedagogía, currículo y didáctica, para comprender los fundamentos pedagógicos de las carreras en estudio. Luego, en un segundo momento, se revisan estos conceptos a la luz de tres enfoques pedagógicos promoviendo que, quien lee, se identifique con alguno de estos enfoques, según sea su interés docente y su aplicación práctica.

Palabras Clave: Pedagogía, Educación Superior, Currículo, Didáctica.

\section{ABSTRACT:}

In the framework of the research entitled Relating pedagogy, curriculum and didactics: the case of the majors offered by the University of Costa Rica, reaccredited by SINAES, this essay shares some theoretical reflections from a Iberoamerican perspective, enriched with the contributions of people interviewed, who coordinate or lead projects and programs directly linked to the teaching faculty of the University of Costa Rica with the purpose of promoting the improvement of teaching performance. The essay is structured in two parts: the first part is a reflection on education, pedagogy, curriculum and didactics in order to understand the pedagogical bases of the majors under study. In the second part, these concepts are reviewed in the light of three pedagogical approaches with the idea of promoting that faculty members identify themselves with one of these approaches according to their teaching interests and practical application.

KEYWORDS: pedagogy, higher education, curriculum, didactics.

\section{INTRODUCCIÓN: INICIANDO LA REFLEXIÓN}

Al reflexionar sobre pedagogía, educación, currículo y didáctica se hace necesario iniciar con un análisis del concepto pedagogía, su nacimiento, su evolución y su significado actual, para continuar luego con los otros tres conceptos: educación, currículo y didáctica, pues constituyen parte del objeto de estudio de la pedagogía. Es así que, en el contexto de la Educación Superior, y particularmente el de la Universidad de Costa Rica, referirse a la pedagogía significa mirar sus procesos educativos, las distintas propuestas curriculares y las estrategias didácticas que desarrolla, por consiguiente es, a través de ellas, que cumple con su Mandato Pedagógico Institucional (González, 2017).

Tomando en cuenta que la pedagogía tiene por objeto de estudio al fenómeno educativo intencionado, y que este fenómeno educativo se desarrolla con el fin de formar un determinado tipo de ser humano, que

\section{NotAS DE AUTOR}

1 Máster en Psicopedagogía. Docente Catedrática de la Universidad de Costa Rica. Docente, investigadora y asesora en diseño curricular e innovación docente. Labora en el Instituto de Investigaciones en Educación, en la Sede del Atlántico y en la Escuela de Geografía 
haya aprendido/construido determinados conocimientos, desarrollado determinadas habilidades y destrezas y que se caracterice por determinadas actitudes y valores, se puede afirmar que el currículo, por su parte, es una estructura o instrumento creado para promover acciones en pro de la formación de ese tipo de ser humano, tradicionalmente denominado perfil, y que se refiere específicamente a "la descripción clara del conjunto de capacidades y competencias que identifican la formación de una persona para encarar responsablemente las funciones y tareas de una determinada profesión o trabajo". (Hawes y Corvalán, 2005, p. 13)

En el caso de la Universidad de Costa Rica, este perfil profesional de egreso es definido para cada una de las propuestas curriculares existentes (carreras), previamente aprobadas por el Centro de Evaluación Académica (en adelante CEA), y se pone en práctica ejecutadas en los distintos espacios de aprendizaje, usualmente denominados cursos, del plan de estudios. Estos cursos son, por excelencia, el espacio de interacción docente - estudiantes, de manera tal que, en estos espacios, se logra, generar las oportunidades de formación educativa para que la población estudiantil construya conocimientos, desarrolle habilidades y destrezas, y se apropie de determinadas actitudes y valores.

Dicho de otro modo, es en los cursos, o como se expresa tradicionalmente, en las aulas, que se concreta el fin de cualquier institución educativa. Es allí donde, como docentes, se intenta educar/formar a la población estudiantil con base en el programa del curso, que, a su vez, se fundamenta en el perfil de egreso de la profesión o carrera en la que se trabaja y, a su vez, este perfil profesional responde al Mandato Pedagógico Institucional o perfil de salida de la persona graduada de la institución en que se labora.

Conociendo que los procesos educativos, o experiencias educativas, no son neutros, sino que son dirigidos, orientados y determinados por factores, como la formación y profesión de las personas que en ellos participan, es posible deducir que estos procesos educativos están influidos por esas personas, por sus actitudes, valores, habilidades, informaciones, ideas, criterios, acerca de sí mismos y del mundo en que interactúan. Todo lo anterior, en conjunto, constituye el quehacer que orienta la formación del perfil de ser humano determinado por cualquier institución educativa.

El objetivo de este ensayo es proponer a la comunidad académica de la Universidad de Costa Rica un concepto de coherencia curricular, no solo a partir de la teoría, sino también a partir del criterio de personas que dirigen, coordinan o lideran distintos espacios para la mejora académica y docente, todo desde una perspectiva hispanoamericana y sobre todo latinoamericana, con el propósito de visualizar las nuevas voces de nuestro continente y contribuir con la producción teórica autóctona. Esta mezcla, teoría y criterios regionales sobre la experiencia, promoverá su comprensión y una mejor adaptación en el contexto institucional de esta Universidad.

Para lograrlo, el presente trabajo se estructura en dos partes fundamentales. La primera parte es la reflexión teórica acerca de los conceptos Pedagogía, Educación, Currículo y Didáctica, a partir de aportes de autores de países latinoamericanos y españoles y de las reflexiones empíricas de docentes universitarios de la Universidad de Costa Rica, que en el momento de la entrevista, coordinaban distintos proyectos o instancias de la Vicerrectoría de Docencia, como la dirección del Centro de Evaluación Académica y las coordinaciones de RIFED, METICS, DEDUN, Proyecto Multiversa y Proyectos de Docencia.

La segunda parte de este trabajo consiste en una propuesta para revisar la coherencia entre la educación, el currículo y la didáctica. Tarea que es compleja, pues, como se señaló líneas antes, influye la visión de cada una de las personas que participan en estos procesos educativos, que simultáneamente aprenden y enseñan, y que asumen una posición personal en cuanto a la definición de estos conceptos. Este instrumento puede constituirse en un insumo, el cual utilicen las distintas Unidades Académicas de la Universidad de Costa Rica, para la reflexión de sus propios procesos curriculares.

Con este ensayo se ofrece, fundamentalmente, insumos teóricos autóctonos que guíen las acciones pedagógicas, curriculares y didácticas de la comunidad académica de la Universidad de Costa Rica y una vía para lograr la coherencia curricular. 


\section{Pedagogía}

El concepto pedagogía no existe aislado, sino que se comprende en contexto, según su intencionalidad específica. En el momento actual existe un debate entre varias posturas, pues se plantea que la pedagogía es, entre otras cosas, un arte, una ciencia, una metodología o una didáctica. En el presente ensayo se asume a la pedagogía como ciencia cuyo objeto de estudio es el fenómeno educativo que, en este caso en particular, es el acto educativo universitario -Educación Superior-, específicamente, en el ámbito curricular y en el respectivo ejercicio didáctico.

La pedagogía es todo ese escenario ... es cómo yo me relaciono con el otro. Ese escenario que se construye para un aprendizaje conjunto, articulado con el contexto y con la realidad de la persona, del estudiante y del profesor y que podamos construir, a partir de ahí, el acto de construcción colectiva en una relación de respeto, de crecimiento de ambos ... es la misma persona en sí misma en su acto de compartir o de construir. (Marta Picado Mesén ${ }^{2}$, comunicación personal, 7 setiembre 2016)

Existe una diferencia entre pedagogía y educación. Los actos educativos son actos naturales, espontáneos y se presentan en todos los ámbitos de la vida. Mientras que la pedagogía se refiere a actos educativos intencionados, pensados, dirigidos al logro de determinados objetivos y relacionados, siempre, con el desarrollo de procesos de aprendizaje y experiencia de las personas participantes y que "implica actos deliberados y aspiraciones de ser humano”. (Eleonora Badilla Saxe ${ }^{3}$, comunicación personal, 30 de abril 2016; Flora Salas Madriz ${ }^{4}$, comunicación personal, 20 abril 2016). En este caso, la pedagogía, o los actos pedagógicos, no estudian, necesariamente, todos los aprendizajes en todos los ámbitos de la vida, ni en todos los fenómenos educativos, sino que se focaliza en aquellos ubicados en un contexto determinado, en el cual se llevan a cabo estas acciones, es decir, en contextos educativos, sean formales o no formales. En este sentido, es posible sostener que uno de los temas que estudia la pedagogía son los procesos educativos concretados mediante propuestas curriculares y sus respectivas estrategias didácticas.

La pedagogía, por lo tanto, es un quehacer humano, el cual ha evolucionado en el tiempo, para constituirse, hoy en día, en una ciencia con su propio objeto de estudio, el cual es, a criterio de Patricia Marín Sánchez ${ }^{5}$ (comunicación personal, 24 junio, 2016), "el proceso formativo que tiene que ver con todas las intencionalidades que tiene la sociedad, y cómo se materializan en el proyecto educativo" y, a partir de un trabajo metódico, produce lo que se suele llamar Teoría educativa, es decir, saberes teórico-técnicos aplicables en diferentes quehaceres educativos que orientan el logro de esa intencionalidad pedagógica (González, 2014; Aldana, 1996), dándole "el cuerpo de conocimiento que sustenta la acción educativa deliberada". (Eleonora Badilla Saxe, comunicación personal, 30 de abril 2016, Flora Salas Madriz, comunicación personal, 20 abril 2016).

Llamamos pedagogía a la reflexión sistemática sobre la educación. La pedagogía es la ciencia de la educación; por ella adquiere unidad y elevación la acción educativa. La educación sin pedagogía, sin reflexión metódica, sería pura actividad mecánica, mera rutina. La pedagogía es una ciencia del espíritu, y está en íntima relación con la filosofía, la psicología, la sociología y otras disciplinas, aunque no depende de ellas, ya que es una ciencia autónoma. La educación y la pedagogía están en la relación de la práctica y la teoría, de la realidad y la idealidad, de la experiencia y el pensamiento, pero no como entidades independientes sino fundidas en una unidad indivisible como el anverso y el reverso de una moneda. (Luzuriaga, 1962, p. 8)

La pedagogía es una ciencia dinámica en continuo movimiento, debido a que es un acto meramente humano. Este quehacer pedagógico se orienta a partir de presiones, fuerzas y necesidades contextuales que determinan líneas de formación humana por parte de los gremios educativos y otros actores sociales, como el mercado o las tecnologías de la información y comunicación digital, por lo que son redefinidas en cada época en función de determinado tipo de sociedad, asociadas a un determinado modo de producción y una forma cultural propia de entender el mundo. Esta ciencia, además, tiene carácter de interdisciplinariedad, pues retoma aportes de otras ciencias básicas y sociales. Al respecto, Aldana (1996) lo sintetiza al declarar que 
La pedagogía se encarga de entender y transformar un objeto de estudio bien determinado (la educación) pero solo desde los condicionamientos y caracterizaciones que la realidad le hace o provoca a dicho objeto de estudio. (p. 20)

La pedagogía es descripción, regulación y transformación del hecho educativo. Pero no de manera exclusivamente reflexiva, sino desde prácticas y reflexiones sistematizadas que van otorgando cientificidad a los planteamientos pedagógicos. Es la ciencia que se ocupa del estudio y transformación de la educación (p.41).

Luzuriaga (1962) coincide con esta visión, pues piensa que, aunque la educación es un elemento esencial y permanente de la vida individual y social, no se ha realizado siempre del mismo modo, sino que ha variado conforme a las necesidades y aspiraciones de cada pueblo y de cada época. La sociedad a que se refiere la educación no es, en efecto, algo estático, constituido de una vez para siempre, sino que está en continuo cambio y desarrollo.

La pedagogía, como quehacer, tiene aproximadamente trescientos años (Aldana, 1996) y como concepto nace relacionado con la niñez, pues -etimológicamente- significa conducción del niño (vocablos griegos: pais, paidos = niño; agoo, agogía = conduzco, conducir), y se aplicaba al quehacer de los esclavos que cuidaban niños, pues eran quienes estaban a cargo del cuidado de "los niños de su amo: los llevaba o conducía a todos los lugares a los que debían ir, como la palestra, el teatro. Cuidaban que esos niños se comportaran según las costumbres que ellos consideraban convenientes a sus conceptos morales" (Aldana, 1996, p. 28).

Por eso, hoy en día, existen quienes se oponen a ser llamados pedagogos y proponen actualizar el concepto, para que se les denomine teóricos de la educación. Al respecto, la reflexión de Flora Salas Madriz es aclaradora cuando comenta:

La palabra 'pedagogía' etimológicamente fue construida en un momento histórico particular y con un sentido específico. Ha sido una palabra que se ha tratado de dejar de usar en ciertos sectores de la comunidad científica en educación o de profesionales de la educación, porque se queda sumamente corta en relación con lo que es el fenómeno educativo como lo conocemos hoy. Desde este punto de vista, pedagogía sería la abuelita de la teoría de la educación. ...Cuando decimos pedagogía significamos teoría de la educación, la pedagogía vendría a ser el equivalente a la teoría de la educación contemporánea (comunicación personal, 20 abril 2016)

Esta idea de pedagogía, como concepto que fue evolucionando hasta llegar a la figura actual y que concibe al pedagogo, o pedagoga, como la persona que se "dedica al estudio, propuesta y conducción técnica de la educación” (Aldana, 1996, p. 28), incluye tanto a docentes como a investigadores o teóricos de la educación. El concepto ha experimentado distintas etapas en su proceso evolutivo, concebido, inicialmente, como una actividad humana espontánea, hasta consolidarse, hoy en día, como una ciencia. En una primera etapa, la filosófica, al iniciarse los primeros planteamientos reflexivos, limitados a la comprensión filosófica "ningún planteamiento pedagógico se hacía sin basarse en una concepción determinada del ser humano" (Aldana, 1996, p. 50). La segunda etapa, la científica, se desarrolla en el siglo XVIII, iniciando en 1774, cuando la "Universidad alemana obliga a sus profesores a enseñar pedagogía. Es decir, a reflexionar sobre la educación" (Aldana, 1996, p. 50) y se gesta la idea de una teoría de la educación. Con la obra La Pedagogía General Derivada del Fin de la Educación de Herbart, en 1806, se marca el punto de referencia de la pedagogía como ciencia, con base en "una doble fundamentación: la filosofía y la psicología" (Aldana, 1996, p. 51). En ese momento se teoriza sobre educación y se inician las instituciones científico-pedagógicas, con especialización universitaria. "Gracias a Herbart, la pedagogía fue vista como una ciencia, y además, como un saber autónomo, no formando parte de otras disciplina (como la filosofía, la sociología, la biología o la psicología)" (Aldana, 1996, p. 52). En la tercera etapa, llamada por Debesse, en el año 1967, ciencias de la educación y cuyos antecedentes se ubican a partir de 1912, con la creación del Instituto de Ciencias de la Educación J.J. Rousseau, se afirmó que la pedagogía "no se trata de una ciencia particular dedicada al estudio de la educación sino que esta constituye una realidad que puede -y debe- ser estudiada por diferentes ciencias... dándole un interés pluridisciplinario" (Aldana, 1996, p. 52) que se enfoca en tres ámbitos: el estudio del problema educativo, el sujeto de la educación y la didáctica. A criterio de Aldana (1996), la pedagogía es autónoma en relación con su objeto de estudio -la educación-; pues tiene la posibilidad de obtener, desarrollar, 
organizar y plantear conocimientos sobre la realidad educativa a partir de la conjunción de dos fuentes de trabajo: la empírico-experimental y la analítica de sistemas y configuraciones de valores (Aldana, 1996).

\begin{abstract}
La teoría de la educación padece del mismo fenómeno que ha ocurrido en las ciencias sociales. Hereda una tradición precientífica, ejercida, sobre todo, por personas del área de la filosofía y más ideólogos que teóricos de la educación. Esta gran tradición pre-científica viene desde la antigua Grecia clásica, con la 'Paideia', que atraviesa toda una serie de posiciones de pensamiento, en donde la educación pasa a ser un recurso para socializar, de cierto modo, a las poblaciones jóvenes. Se creía que las sociedades se podían construir siguiendo un esquema y modelo a través de la educación e instruir a la gente para que se adaptara y formara de acuerdo con eso, y cuyo resultado fuera una persona competente y funcional en esa sociedad. En ese momento ni siquiera había universidad, estaba la academia, estaba el liceo y se educaba cierta gente que podía acceder a esos procesos, pero, típicamente, la educación no era una necesidad de toda la sociedad, sino de una minoría. La formación se convierte en el músculo de lo ideológico en el periodo medieval, cuando la educación es educación religiosa. Músculo profundo, fuerte y dirigido a sectores privilegiados de la población. Por eso, la educación, literalmente, era memorice, oiga, crea y punto, era un acto de fe. La educación pasa de ser músculo para la construcción de imperios a ser músculo para la ideologización y el dogma religioso hasta la Edad Media. Ya en la Edad Media, hay un salto histórico cualitativo, que no solo afectó a la educación, sino todos los demás ámbitos de la vida. (Flora Salas Madriz, comunicación personal, 20 abril 2016).
\end{abstract}

Como se argumentó en líneas anteriores, en este ensayo se asume el concepto de pedagogía como ciencia, cuyo objeto de estudio es el acto educativo, mediante un método de investigación riguroso, esencialmente interpretativo, experimental y analítico, que genera teoría educativa. Se espera que esta reflexión pedagógica sirva de base, de sustento, de fundamento y de orientación al proyecto educativo de la Universidad de Costa Rica, así como al análisis de sus programas curriculares que, dirigidos a la formación de seres humanos, en respuesta a una determinada visión de mundo y a un determinado tipo de ser humano, se concretan con las estrategias didácticas aplicadas en los distintos cursos impartidos.

\title{
Educación
}

Si la pedagogía es la ciencia que tiene por objeto de estudio a la educación, entonces puede definirse como el proceso experimentado por los seres humanos durante su vida. Es el proceso, espontáneo y social, determinado por el contexto y por las características de personalidad, en que se desarrollan estos seres y, mediante el cual, comprenden su entorno, aprenden a comunicarse (señas, lenguaje, idioma) y a desenvolverse, interactúan, construyen herramientas para transformar su cultura, sienten, piensan, aman, es decir, se realizan como seres. La pedagogía estudia, por lo tanto, todos estos procesos, en relación con sus procesos de aprendizaje asociados.

La educación, como proceso consciente o inconsciente, tiene la finalidad de compartir con el otro, y consigo mismo, la experiencia para comprender el mundo. Freire (1997) mantiene que "la educación verdadera es praxis, reflexión y acción del hombre sobre el mundo para transformarlo" (p. 7) y que "no existe educación sin sociedad humana y no existe hombre fuera de ella” (p. 25). Luzuriaga (1962), por su parte, expresa que la educación es

La influencia intencional y sistemática sobre el ser juvenil con el propósito de formarlo o desarrollarlo. Pero también significa la acción general, difusa, de una sociedad sobre las generaciones jóvenes con el fin de, conservar y transmitir su existencia colectiva. La educación es así una parte integrante, esencial, de la vida del hombre, y de la sociedad, y ha existido desde que hay seres humanos sobre la tierra (p. 7).

Sin embargo, también se observa en la literatura que, a partir de la Edad Media, se atribuye a la educación un carácter formal, intencionado y estructurado con el fin de enseñar la cultura a los otros, por lo que merece la pena hacer una distinción entre un acto educativo y un acto pedagógico. Un acto educativo es un acto espontáneo en el cual participan los seres humanos, en una constante y continua interacción social, mientras que un acto pedagógico responde a un propósito, tiene una intención definida y dirigida a la formación de otras personas, con determinadas características deseadas por quienes lideran esos procesos pedagógicos. 
En otras palabras, el acto pedagógico lleva implícito el aprendizaje y su evaluación, mientras que los actos educativos no necesitan ser evaluados por el otro para emitir un juicio de valor.

La educación es un componente tan fundamental de la cultura, como puedan serlo la ciencia, el arte o la literatura. Sin la educación no sería posible la adquisición y transmisión de la cultura, por ella vive ésta en el espíritu de los hombres. Una cultura sin educación sería una cultura muerta. Y ésta es también una de las funciones esenciales de la educación, hacer que la cultura siga viviendo a través de los siglos (Luzuriaga, 1962, p. 7).

Educación es, de alguna manera, el acto humano de intercambio de saberes, natural, espontáneo, vital y social, entendido como un acto que se da en interacción con el otro, fuera de uno, es decir, entre y con otros seres humanos y en interacción constante con el contexto. Es mediante este acto que los seres humanos enseñan y aprenden sobre su mundo para comprenderlo y vivir en él. "La educación está relacionada con lo común, con la comunidad y con la comunicación. Posee una función social e implica crecimiento, dirección y control”. (Trilla, Cano, Carretero, Escofet, Fairstein, Fernández y Vila, 2001, p. 25)

Antes de la consolidación de la época industrial, la educación era más orgánica, menos programada, más natural y espontánea. Mientras que cuando se consolida la Revolución Industrial, las sociedades -sus grupos de poder político y económico- se enfrentan al desafío de socializar el conocimiento necesario para involucrar trabajadores-obreros en sus procesos industriales por lo que les necesitaban con cierto nivel de conocimiento para que pudieran utilizar los instrumentos, las técnicas y las máquinas requeridas en los procesos industriales. De manera tal que se hacía necesario generalizar las habilidades de lectura y escritura. Es en ese momento que los procesos educativos adquieren otras características, convirtiéndose en un acto planificado, intencionado y dirigido a la enseñanza de determinados conocimientos. Allí nacen las raíces de la pedagogía, transformándose, radicalmente, la visión sobre la educación, pues adquiere un carácter instructivo, con una intencionalidad política, en aras de fortalecer el sistema organizativo de la cultura y de transmitir los conocimientos construidos a partir de la visión mecánica de mundo, que era la prevaleciente en esa época. Una visión que, según Capra (1991), surge en el siglo XVII a partir de los aportes de Descartes y Newton, y que se asocia con un sistema de valores que deja de mirar al mundo como algo vivo y verlo como un objeto.

La visión mecanicista del mundo la desarrollaron en el siglo XVII Galileo, Descartes, Bacon y Newton, entre otros. ... El universo material, incluyendo el organismo humano, era una máquina que, en principio, podía entenderse completamente con sólo analizar separadamente sus partes más pequeñas. ... Antes de la revolución científica de Galileo, Descartes, Bacon, Newton, la ciencia tenía como objeto la sabiduría, la comprensión del orden natural y el vivir en armonía con ese orden. A partir del siglo XVII, el objeto de la ciencia ha sido el conocimiento que pueda emplearse para controlar, manipular, explotar la naturaleza. (Capra, 1991, pp. 28-29).

Este fenómeno tuvo fuerte impacto en la sociedad y sus procesos formadores, pues a partir de ese momento, el ser humano pasó de ser un ser vivo, con sus propios ritmos naturales, a ser un objeto vivo que necesitaba formarse -o, más exactamente, instruirse- con los conocimientos -informaciones- que se le entregaban y que eran construidos a partir de la investigación científica, pues eran los legitimados socialmente. La educación, por lo tanto, comienza a ser un proceso político, masivo y con la intención fundamental de enseñar a leer y escribir, para leer los conocimientos, instrucciones y lineamientos seleccionados por quienes ejecutaban los procesos educativos. En palabras de Illich, se crea la institución escolar que "enseña la necesidad de ser enseñado" (Trilla et ál., 2001, p. 282) y de Savater (2016), al reiterar que "educar no es formar empleados, sino crear ciudadanos" (párr. 11).

Actualmente transitamos el ocaso de la Revolución Industrial hacia una nueva época llamada Informacionalismo (Castells, 2002; Capra, 1992, Souza, 2011). En este proceso, quienes ejercen la educación continúan considerando, en términos generales, que la prioridad es transmitir conocimientos. Sin embargo, actualmente se experimentan transformaciones cualitativas en el contexto, que obligan a reavivar el rol de la educación como proceso de formación de seres humanos que sepan pensar complejamente, más allá de la memorización de información y la capacidad técnica. 
Debemos trazar una distinción clara entre la inteligencia humana y la inteligencia de una máquina. La inteligencia humana, los juicios humanos, la memoria humana y las decisiones humanas nunca son completamente racionales, sino que están siempre teñidas por las emociones. Nunca podemos separar la racionalidad humana de la emoción, ni tampoco de la intuición. Por otra parte, nuestro pensamiento se ve siempre acompañado de sensaciones y procesos corporales. ... La visión mecanicista y fragmentada es una característica básica de la caduca visión del mundo. Otra es la obsesión por dominar y controlar. ... Nuestra ciencia y nuestra tecnología se basan en la creencia de que la comprensión de la naturaleza implica el dominio de la naturaleza por parte del hombre.... (Capra, 1991, p. 29)

Bajo esta tesitura, lo importante, por lo tanto, es, en primer lugar, comprender y aceptar que la educación ha pasado de ser únicamente un proceso natural espontáneo, para ser, adicionalmente, un proceso intencionado y estructurado por personas con poder y con determinados objetivos sociales, humanos, económicos, ...; que, además, está legitimado y aceptado socialmente. En segundo lugar, es necesario asumir que los desafíos educativos de la sociedad actual trascienden la transmisión de conocimientos. Y, en tercer lugar, aceptar que la formación del ser humano está influenciada por muchos factores relacionados con el cambio de época que experimentamos, el cual afecta todos los ámbitos de la vida, y ha promovido que las personas sean conscientes de su derecho a tomar decisiones sobre su vida y su destino, a pensar complejamente, más allá de memorizar contenidos, comprendiéndolos, utilizándolos y adaptándolos a las condiciones contextuales.

$\mathrm{Al}$ respecto, las palabras de Aldana (1996) son claras y precisas. "La educación es un hecho real, algo que existe, mientras que la pedagogía es el estudio de ese hecho real, de eso que existe”. (p, 19), por lo que para comprender y para crear un fenómeno educativo intencionado, es necesaria la pedagogía.

\section{Currículo}

Por lo tanto, si la educación es un proceso natural, mediante el cual las personas aprenden a vivir, y convivir, en el contexto, gracias a códigos sociales de comunicación, actuación y transformación del contexto, formándose con determinadas características; y si la pedagogía es la ciencia que estudia ese fenómeno para construir teoría educativa que oriente las decisiones educativas; entonces el currículo es la acción humana que estructura, de manera creativa, las acciones planificadas para formar un determinado tipo de ser humano mediante procesos educativos dirigidos al respecto. "El primer momento de la formulación de la teoría curricular se produjo en la gestación de la industrialización monopólica de este siglo” (Bobbit, 1918, Charter, 1924, citados por Díaz, 2010, p. 19) y "se refiere a todo el ámbito de experiencias, de fenómenos educativos y de problemas prácticos, donde el profesorado ejerce su oficio y el alumnado vive su experiencia escolar" (Escudero, 1999, p, 28)

En su origen, el currículo significó el territorio acotado y regulado del conocimiento que representa los contenidos que el profesorado y los centros educativos tendrán que desarrollar; es decir, el plan de estudio propuesto e impuesto en la escolaridad a profesores (para que lo enseñen) y a estudiantes (para que lo aprendan). De todo lo que sabemos y es potencialmente enseñable y posible de aprender, el currículo es una selección regulada de los contenidos a enseñar y aprender que, a su vez, regulará la práctica didáctica que se desarrolla durante la escolaridad. En la Edad Media el currículo se compone de una clasificación del conocimiento integrado por el 'trivium' (tres caminos o cursos: Gramática, Retórica y Dialéctica), que hoy llamaríamos instrumentales, y el 'cuadrivium' (cuatro vías: Astronomía, Geometría, Aritmética y Música) que tenían un carácter más marcadamente aplicado. Estas siete artes constituyeron una primera ordenación del conocimiento que perduró durante siglos en las universidades europeas. La distinción entre ambos grupos de saberes significa dos orientaciones en la formación: los que se refieren a los modos de adquirir el conocimiento, por un lado, y las que sirven al hombre para ganar su sustento y con una finalidad más pragmática. (Gimeno, 2010, p.22)

El currículo nace y se mantiene, por lo tanto, como una construcción político-ideológica, que de fondo tiene la intención de formar personas que transformen la sociedad. Al respecto, Gimeno (2010) lo expone claramente, cuando enuncia que, actualmente:

... el currículo tiene una capacidad o un poder inclusivo que nos permite hacer de él un instrumento esencial para hablar, discutir y contrastar nuestras visiones sobre lo que creemos que es la realidad educativa [pues contiene lo que] pretendemos que aprenda el alumnado; en qué deseamos que se convierta y mejore (pp. 11-12) 
A su vez, Aldana (1996) ratifica, citando a Shirley Grundy, que "el currículo es una forma de organizar un conjunto de prácticas educativas humanas" (p. 207) y comparte su propio concepto con las siguientes palabras:

Es la representación simbólica de un sistema compuesto por las realidades ocurrentes y las proyecciones sobre las interacciones entre elementos y dinámicas en una institución o proceso educativo. Es decir, el currículo a través de su expresión elaborada, que podríamos llamar 'diseño curricular', nos presenta lo que existe (elementos materiales, impersonales o personales, procesales, filosofía, objetivos, aspectos jurídicos, históricos...), así como las interacciones que tienen lugar entre esos elementos -componentes estáticos- y los procesos -componentes dinámicos-. (Aldana, 1996, p. 207)

Desde la Vicerrectoría de Docencia de la Universidad de Costa Rica, Flora Salas Madriz (comunicación personal, 20 abril 2016) concibe que "el currículo es un marco de referencia para articular un proceso educativo que contiene el nivel filosófico, el fundamento epistemológico y teórico, y las proyecciones de aplicaciones". Agrega Susan Francis Salazar ${ }^{6}$ (comunicación personal, 8 junio 2016) que es, al mismo tiempo, "una selección de cultura desde los discursos, los lenguajes, los artefactos culturales, por lo tanto es un proyecto político, un proyecto que establece en lo que se quiere trabajar”. Entonces, el currículo, más que un producto, es un proceso pensado en torno al camino que han de recorrer las personas que participan en él, para que, en el trayecto, incidan sobre sí mismas, sobre sus propias características, se formen, aprendan, construyan, fortalezcan y reafirmen sus personalidades, características, criterios a partir de conocimientos construidos, habilidades desarrolladas y valores experimentados durante ese trayecto. De ahí la importancia tan grande que tiene el definir, conscientemente, el perfil de persona que se desea ayudar a formar. En el caso de la Educación Superior, es un camino diseñado para formar profesionales, incluyendo su bagaje personal, emocional, ético y cognitivo y que, con el cual, participarán en las dinámicas sociales y transformarán sus contextos.

El currículo es la construcción de una propuesta curricular, que se hace desde un escenario específico que, por su propia naturaleza, siempre está en construcción, es dinámico y tremendamente político, por lo que tiene que estar totalmente inmerso en los debates contemporáneos relativos al ámbito que se está tratando de incursionar y también tiene que hacer una lectura del mercado. Tiene que hacerlo porque representa un amplio sector en el cual van a ejercer las y los futuros profesionales. ... El currículo no solo es el documento que describe la fundamentación con el referente profesional, socioprofesional, pedagógico, epistemológico, la malla curricular y su estructura. No. También están estudiantes, docentes, escenario o escenarios, está el vínculo con la sociedad y están las presiones sociales. (Marta Picado Mesén, comunicación personal, 7 setiembre 2016)

Un posible primer paso, para concretar este camino, o ruta curricular, es el definir las características de la persona-profesional "que se desea formar integralmente conforme a un imaginario deliberado al que llamaremos perfil de ser humano” (Susan Francis Salazar, comunicación personal, 8 junio 2016) y que Bolaños (2015) llama perfil académico de salida con la siguiente definición:

El conjunto de saberes que pretendemos sean desarrollados por los y las estudiantes en el transcurso de la carrera. Se refiere a conocimientos, habilidades y destrezas, así como a aspectos actitudinales y éticos. Se responde a las preguntas: ¿qué debe saber el o la estudiante al concluir el plan de estudios? ¿qué debe saber hacer el o la estudiante al concluir el plan de estudios? ¿cómo debe ser el o la estudiante al concluir el plan de estudios? Las respuestas a estas preguntas son el resultado de la integración de los aportes de los marcos referenciales, a partir de una profunda reflexión en la unidad académica, con la participación de académicos y estudiantes (Bolaños, 2015, p. 6).

Se destaca, en el aporte anterior, que para definir este perfil académico de salida, se hace necesario, previamente, definir unos marcos referenciales, llamados por Bolaños (2015, p.1) “el análisis de la profesión/ práctica, el análisis de la disciplina y el análisis pedagógico”. Es importante que, cuando en las instituciones universitarias, se cree o se revise los perfiles de egreso profesional, se reflexione y describa estos marcos referenciales, pues son fundamentales para crear una propuesta coherente con el Mandato Pedagógico Institucional (González, 2017) y que responda a las necesidades y demandas del contexto. 
Para Bolaños (2015, pp. 5-8) los marcos son tres, el marco socioprofesional, o análisis de la profesión/ práctica, el cual se construye reflexionando en torno al contexto social e histórico de la profesión, mediante la ubicación sociohistórica, el análisis de las prácticas profesionales a nivel institucional, nacional e internacional, la comprensión del estado actual de la profesión y su formación, así como los lineamientos que la unidad académica espera aportar a la sociedad con esa profesión; reflexionando en torno al por qué y para qué se ofrece la carrera y la caracterización de la profesión mediante el análisis de las prácticas profesionales -lo que hacen los profesionales en el campo de trabajo- y la definición profesional -los conocimientos, las habilidades y destrezas y los aspectos éticos y actitudinales- requerida para su desempeño. El marco epistemológico, o análisis de la disciplina, surge a partir de determinar la disciplina, o disciplinas, fundamentales a nivel teórico y metodológico de la profesión, para lo cual se clarifica el objeto de estudio de la profesión, su finalidad y su relación con otras ciencias y disciplinas. También, al revisar su abordaje metodológico, es posible aclarar la forma de producción del conocimiento y su impacto en la realidad profesional y laboral. Y el marco pedagógico, o análisis pedagógico, que se refiere a la definición de las formas en que se desarrollarán los procesos de aprendizaje de la profesión, tomando como punto de partida el concepto que se tenga de aprendizaje y de enseñanza, pues este marco orientará las estrategias didácticas del plan de estudios por diseñar.

Una vez definidos los marcos anteriores, se definen los propósitos de la carrera para continuar con el perfil de salida profesional, definido como "el conjunto de saberes que pretendemos sean desarrollados por los y las estudiantes en el transcurso de la carrera. Se refiere a conocimientos, habilidades y destrezas, así como a aspectos actitudinales y éticos" (Bolaños, 2015, p. 9), o, como lo plantea Ysunza (2010, p. 35), “es, junto con el plan y los programas de estudio, la expresión materializada de una propuesta educativa para la formación de un profesional" $y$

Constituye la base para justificar las decisiones respecto de la estructura y los contenidos del plan de estudios en tanto que expresa de manera sintética y con un mayor nivel de precisión las directrices principales de la propuesta educativa; es decir, el perfil tiene la función de orientar el trabajo subsecuente de diseño curricular y se elabora a partir de dos dimensiones: formativa y profesional. La formativa se refiere a los contenidos académicos: conocimientos, destrezas, habilidades, actitudes y valores requerido para satisfacer las necesidades sociales, profesionales y culturales, mientras que la profesional alude al ejercicio o práctica profesional y está constituida por funciones, tareas, acciones y actividades que será capaz de realizar el egresado en determinadas condiciones de trabajo y campos de desarrollo profesional (Ysunza, 2010, p. 54-55).

Sintetizando, el currículo en la Educación Superior se encarga del diseño de actividades de aprendizaje secuenciadas con el fin de formar-educar personas en función de un determinado perfil de egreso, con una serie de características y conocimientos, llamado comúnmente, perfil de salida, y cuya definición es producto del análisis sociohistórico, epistémico y pedagógico de la profesión que se enseña.

Finalmente, no se debe perder de vista que, en el caso concreto de la Universidad de Costa Rica, el currículo, más allá de "ser de calidad, debe ser de excelencia en primer lugar, porque en él debe darse la integración de la docencia, la investigación y la acción social y, en segundo lugar, tiene que tener una intencionalidad ética y política”. (Marta Picado Mesén, comunicación personal, 7 setiembre 2016)

\section{Didáctica}

La didáctica, por su parte, es el instrumental creativo, en forma de experiencias o actividades, que surge en función del logro del perfil de salida. "Es una forma tecnológica, o sea, es todo el instrumental, todas las técnicas, las herramientas que usted tiene para poner en marcha un proceso formativo" (Susan Francis Salazar, comunicación personal, 8 junio 2016). Es toda actividad creativa, diseñada especialmente para lograr el perfil de salida, tanto en la construcción de conocimientos, como en el desarrollo de habilidades y destrezas, así como de la vivencia de las actitudes y los valores. "No hay ninguna duda de que la didáctica expresa el referente práctico y operativo más natural y propio de la pedagogía”. (Aldana, 1996, p. 219) 
Toda acción didáctica, supone el propósito de mediar, corregir y estimular la experiencia del encuentro entre un sujeto que ejerce una serie de funciones sobre o con un contenido, o mejora capacidades diversas, de suerte que quedan transformadas y enriquecidas dichas funciones y capacidades que en un sentido general reconocemos como aprendizaje. (Gimeno, 2010, p. 37)

Es por eso que se dice que la didáctica es un arte, aunque también incluye técnicas y estrategias. Porque es un poco de todo. Es un arte, pues involucra la dimensión creativa, sin embargo, también tiene un marco de acción, el cual es la disciplina, ciencia, técnicas y métodos necesarios de aprender para lograr el dominio de la profesión que se aprende, por lo cual, ha sido concebida como "el arte de enseñar, de instruir". (Aldana, 1996, p. 220) Esta idea es reforzada por Flora Salas Madriz (comunicación personal, 20 abril 2016) cuando afirma que "la didáctica es muchísimo más pragmática. Es la pragmática educativa, porque se interesa en poner en ejecución un proceso de enseñanza y aprendizaje específico”.

La didáctica es más relacionada con procedimientos, planificación, organización y evaluación, así como del instrumental que se requiera. Es una actividad en la que se confronta mi criterio con el texto, con el estudiantado y con la realidad que estás viviendo. (Marta Picado Mesén, comunicación personal, 7 setiembre 2016)

Este quehacer tiene como génesis el deseo de lograr el éxito en el aprendizaje, y desde esa perspectiva se ha construido teoría didáctica con el fin de guiar a todas aquellas personas que tienen a su cargo tareas educativas. Aldana (1996, p. 222) lo expone claramente: "La didáctica es la referencia a la disciplina pedagógica que se encarga específica y concretamente de estudiar, dirigir y transformar técnicamente los procesos de aprendizaje e instrucción" y que se complementa con el aporte de Patricia Marín Sánchez (comunicación personal, 24 junio, 2016) quien añade que "estudia, analiza y comprende las prácticas didácticas en los escenarios donde se desarrolla el proceso de enseñanza y de aprendizaje”. Sin embargo, poco -o nada- se encuentra ni se escucha, con respecto a la relación entre el perfil de egreso que se desea formar y la teoría curricular y didáctica.

Crear estrategias didácticas para lograr la misión educativa, que se tiene entre manos, depende de tres factores fundamentales. El primero, del Mandato Pedagógico Institucional, es decir, del tipo de ser humano que se desea formar en la institución, organización, ámbito o proyecto educativo en que uno se desenvuelve. El segundo, el perfil de salida de la persona que egresa del proyecto específico de esa institución educativa. Y, el tercero, de su propia posición y enfoque pedagógico que determinan lo que entiende por aprendizaje.

Estos tres factores están unidos por una estructura invisible y es la coherencia que debe existir entre ellos, por lo que la orientación o enfoque didáctico dependerá de la orientación o enfoque curricular y este, a su vez, dependerá del Mandato Pedagógico Institucional. Se puede decir que están unidos por una columna vertebral invisible, a la cual se llamará el enfoque pedagógico. Existe coherencia entre el Mandato Pedagógico Institucional, la propuesta curricular y la didáctica cuando estos tres tienen en sus fundamentos el mismo enfoque pedagógico. Comprender o interpretar esta coherencia es esencial para orientar los esfuerzos a lograrla, reflexión que se aborda en las siguientes líneas.

\section{¿DE QUÉ ESTAMOS HABLANDO AL PLANTEAR LA COHERENCIA ENTRE EDUCACIÓN, CURRÍCULO Y DIDÁCTICA?}

Si la educación tiene como propósito la formación de los seres humanos, si el currículo es un camino para formar esos seres humanos en sus procesos de aprendizaje de conocimientos, habilidades, destrezas, actitudes y valores y si la didáctica es el instrumental creativo que desarrolla estrategias y utiliza determinados recursos para lograr ese aprendizaje propuesto, entonces, lo primero que puede garantizarse es que el eje común, en esta trilogía, es que los tres aspectos se refieren a la formación de un determinado tipo de ser humano o, para llamarlo de una manera más simple, de un perfil de ser humano a formar.

Por lo tanto, para comprender ¿qué se va a entender por coherencia? puede empezarse por el Diccionario de Real Academia Española (2014, párr. 1), que la define como la "conexión, relación o unión de unas cosas con 
otras". En este trabajo se asume la coherencia en esos términos y, por lo tanto, se entiende que la educación, el currículo y la didáctica se cohesionan, se conectan y se relacionan a partir del común denominador, es decir, el ser humano por formar.

Con base en los aportes de las páginas anteriores, es posible sostener que las autoridades universitarias participantes coinciden en que estos tres conceptos tienen una relación directa. "La educación es la visión, la intención; el currículo es el esqueleto, la forma de organizar esa intención y la didáctica es el músculo, la forma de llevar al aula esta intención" (Eleonora Badilla Saxe, comunicación personal, 30 de abril 2016).

La relación de estos tres conceptos es inmediata. No hay manera de que haya una teoría de la educación en donde no exista una conceptualización del currículo y no haya una serie de didácticas o prácticas educativas concretas que se deriven de él, porque ese es el fondo de todas las disciplinas: la articulación entre fundamentación epistemológica, teoría, metodologías de investigación y prácticas o tecnologías. (Flora Salas Madriz, comunicación personal, 20 abril 2016)

Hablando metafóricamente, Susan Francis Salazar (comunicación personal, 8 junio 2016) opina que "la pedagogía formula el camino, el currículo establece las esquinas, las vueltas, los virajes, las rectas, las montañas del camino y la didáctica dice a la persona cómo llevará ese camino”. Por lo tanto, otra afirmación posible, es que no se puede ignorar la relación directa que existe entre educación, currículo y didáctica, así como tampoco la relación de estos conceptos con la pedagogía, pues esta última, en su condición de ciencia, tiene por objeto de estudio, el fenómeno educativo, que los incluye.

Una segunda dimensión en este análisis, es el hecho de que "el amarre de estas categorías se da a partir de un determinado enfoque pedagógico que considera el tipo de ser humano que se desea formar" (Patricia Marín Sánchez, comunicación personal, 24 junio 2016). Esta última reflexión dirige la mirada a un aspecto de fundamental importancia, cuando se habla de coherencia en el marco de un fenómeno tan particular como es el educativo, pues se desarrolla en contextos eminentemente sociales, y es el hecho de tomar en cuenta que, al momento de asumir un determinado perfil de ser humano, se asume, de manera vinculante, un determinado enfoque pedagógico.

Continuando con los aportes del Diccionario de la Real Academia Española (2014, párr. 1), se observa que enfoque es la "acción y efecto de enfocar", o dicho de otro modo, es dirigir la atención o el interés hacia un asunto o problema con base en ideas preconcebidas, lo cual significa, proyectado a la docencia, que cada docente, en el ejercicio de sus labores, pone atención a su quehacer desde determinadas ideas previas acerca del aprendizaje que caracterizan su visión de mundo, lo que la acerca a uno -o varios- enfoques pedagógicos determinados.

Cobra importancia, en este momento, recordar a De Souza Silva (2011, p. 104) cuando propone que el planeta tierra está experimentando la convergencia de, al menos, tres revoluciones en conflicto, las cuales pueden ser denominadas: la revolución cibernética, la revolución económica y la revolución contextual de mundo, y que cada una lleva implícita una determinada visión de mundo que, a su vez, se refiere a un determinado perfil de ser humano (Tabla 1). 


\begin{tabular}{|c|c|c|}
\hline Visión cibernética & Visión mercadológica & Visión contextual \\
\hline $\begin{array}{l}\text { Metáfora guía: el mundo } \\
\text { es una máquina que } \\
\text { funciona como un sistema } \\
\text { de información } \\
\text { autorregulado; un mundo } \\
\text { constituido de redes } \\
\text { cibernéticas, donde todo } \\
\text { es reducido a información } \\
\text { y todos son percibidos } \\
\text { como consumidores, } \\
\text { procesadores y } \\
\text { productores de } \\
\text { información. }\end{array}$ & $\begin{array}{l}\text { Metáfora guía: el mundo } \\
\text { es un mercado, un } \\
\text { conjunto de arenas } \\
\text { comerciales y tecnológicas } \\
\text { cuya misión es la función } \\
\text { económica. Las personas, y } \\
\text { la naturaleza, son } \\
\text { proveedores, productores, } \\
\text { clientes, procesadores, } \\
\text { competidores, } \\
\text { inversionistas, } \\
\text { consumidores, } \\
\text { exportadores, pues todo } \\
\text { puede ser vendido y } \\
\text { comprado. }\end{array}$ & $\begin{array}{l}\text { Metáfora guía el mundo es } \\
\text { una trama de relaciones y } \\
\text { significados entre } \\
\text { diferentes formas y modos } \\
\text { de vida, con amenazas } \\
\text { creadas por la acción } \\
\text { humana y cuya solución } \\
\text { depende de que la } \\
\text { sostenibilidad sea percibida } \\
\text { como una propiedad } \\
\text { emergente de la interacción } \\
\text { humana para superar } \\
\text { nuestra vulnerabilidad. }\end{array}$ \\
\hline $\begin{array}{l}\text { Los seres huma } \\
\text { recursos huma } \\
\text { piezas del engra } \\
\text { porque todo lo } \\
\text { en la máquina e } \\
\text { percibido como } \\
\text { recursos natura } \\
\text { recursos financi } \\
\text { recursos human }\end{array}$ & $\begin{array}{l}\text { Los seres humanos son } \\
\text { capital humano o capital } \\
\text { intelectual, porque todo lo } \\
\text { que entra en el mercado es } \\
\text { percibido como capital: } \\
\text { capital natural, capital } \\
\text { financiero, capital social, } \\
\text { capital humano, etc. }\end{array}$ & $\begin{array}{l}\text { Los seres humanos son } \\
\text { talentos humanos; el } \\
\text { mundo tiene } \\
\text { potencialidades naturales, } \\
\text { humanas, etc. Nuestra } \\
\text { imaginación nos permite } \\
\text { crear más allá de la } \\
\text { experiencia actual y del } \\
\text { conocimiento previo. }\end{array}$ \\
\hline $\begin{array}{l}\text { Las organizaciones son } \\
\text { máquinas innovadoras } \\
\text { que consumen, procesan } \\
\text { y producen información, } \\
\text { que es transformada en } \\
\text { bienes y servicios a ser } \\
\text { ofertados. La } \\
\text { organización sostenible es } \\
\text { la organización eficiente; } \\
\text { a mayor grado de } \\
\text { eficiencia mayor grado de } \\
\text { sostenibilidad. La } \\
\text { eficiencia productiva es } \\
\text { Su objetivo. }\end{array}$ & $\begin{array}{l}\text { Las organizaciones son } \\
\text { proveedores de bienes y } \\
\text { servicios demandados por } \\
\text { el mercado, que es la } \\
\text { principal fuente de } \\
\text { referencia para la } \\
\text { innovación. La } \\
\text { organización sostenible es } \\
\text { la organización } \\
\text { competitiva; a mayor grado } \\
\text { de competitividad mayor } \\
\text { grado de sostenibilidad. La } \\
\text { mayor competitividad es su } \\
\text { objetivo. }\end{array}$ & $\begin{array}{l}\text { Las organizaciones son } \\
\text { facilitadores de cambio, } \\
\text { inspiradas en los desafíos } \\
\text { (necesidades, realidades y } \\
\text { aspiraciones) del contexto } \\
\text { donde ocurre la aplicación e } \\
\text { implicaciones de sus } \\
\text { contribuciones. La } \\
\text { organización sostenible es } \\
\text { la organización cambiante, } \\
\text { que innova y cambia junto } \\
\text { con su entorno cambiante. }\end{array}$ \\
\hline
\end{tabular}

Fuente: De Souza Silva, 2011.

De manera tal que, buscar la coherencia, es decir, buscar el enfoque pedagógico presente en el Mandato Pedagógico Institucional, así como en sus distintas propuestas curriculares y didácticas, puede iniciarse descubriendo o reconociendo cuál visión de mundo subyace en ese perfil de ser humano, para luego encontrar el enfoque pedagógico que responde a cada una de estas visiones de mundo, y, con ello, detectar, con base en distintos enfoques curriculares, cuál de ellos es coherente con el Mandato Pedagógico Institucional. El mismo ejercicio puede hacerse con el tema didáctico.

Partiendo de estas tres visiones de mundo, podría pensarse, en términos pedagógicos, que la teoría educativa responde a una de estas tres visiones de mundo, para lo cual se retoman los aportes de González (2014, p. 58) cuando plantea que cada docente puede "reconocer su propio enfoque pedagógico, reconocer el enfoque pedagógico de la propuesta curricular y, algo mucho más importante, si su enfoque pedagógico personal es diferente al enfoque de la carrera en que participa". La autora propone tres enfoques pedagógicos que denomina conductista, porque el aprendizaje se orienta a la memorización de informaciones como sinónimo de conocimientos, cognitivo, porque se promueve la construcción del aprendizaje mediante un rol 
activo del estudiantado y crítico, porque el aprendizaje se construye cuando la información interactúa con alguna realidad determinada, en un diálogo permanente.

De igual manera, es posible encontrar enfoques curriculares. Tal es el caso de la propuesta de Ruiz (2005), quien plantea tres: tecnológico, deliberativo y crítico o Escudero (1999) quien plantea el técnico y experto; el deliberativo y práctico y el crítico y postmoderno. Puede inferirse, a partir de las explicaciones de estos autores, que el enfoque tecnológico y el técnico y experto responden a las mismas premisas pedagógicas, pues ambas plantean que la realidad es estática, única, convergente, fragmentable y que se comprende a partir del método científico, que el conocimiento curricular es universal, objetivo, neutro, racional, controlable y eficaz. Por otra parte, puede establecerse una relación entre el enfoque deliberativo y práctico, pues ambas conciben la realidad con un fenómeno dinámico, múltiple, holístico y divergente, en tanto el currículo se construye con un fin "estrictamente técnico" (Escudero, 1999, p. 125) y práctico o experiencial, es decir, promoviendo experiencias de aprendizaje colaborativas, lo que le da el carácter de deliberación con énfasis en la construcción de conocimiento. Finalmente, el enfoque crítico se relaciona con el crítico y postmoderno, dado que conciben la realidad como un fenómeno compartido, histórico, construido dinámica y colectivamente y que la construcción del conocimiento se realiza desde la participación y comunicación, influenciados por el contexto (cultura, política, economía, otros) cuyo conocimiento curricular busca identificar y comprender los problemas en su contexto en un diálogo teoría-realidad. Se asume, en el contexto de este ensayo, las denominaciones de Ruiz (2005) tomando en cuenta que, para el análisis, serán considerados los aportes de Escudero (1999).

Volviendo a la pregunta inicial: ¿De qué estamos hablando al plantear la coherencia entre educación, currículo y didáctica? Se propone la siguiente respuesta. La coherencia entre educación, currículo y didáctica se encuentra cuando, en una propuesta curricular determinada, todos sus elementos responden al mismo -o mismos- enfoques pedagógicos, pues puede darse el caso de que se asuma premisas pedagógicas de distintos enfoques pedagógicos. En este sentido, el propósito de encontrar la coherencia, no es dictaminar qué enfoque debe prevalecer en cada propuesta curricular, sino que las personas involucradas en esta propuesta curricular puedan encontrar el enfoque pedagógico tanto en su perfil de egreso como en su propuesta curricular y en las estrategias didácticas que desarrolla, tanto para determinar su coherencia interna como para su correspondencia con el Mandato Pedagógico Institucional. 
La Tabla 2. muestra una guía que puede servir de instrumento para detectar la coherencia entre los enfoques.

\begin{tabular}{|l|l|l|l|}
\hline \multicolumn{3}{|c|}{ Tabla 2: Coherencia entre pedagogía, educación, currículo y } \\
didáctica \\
\hline $\begin{array}{c}\text { Enfoque } \\
\text { pedagógico }\end{array}$ & $\begin{array}{c}\text { Educación } \\
\text { visión de } \\
\text { mundo - ser } \\
\text { humano }\end{array}$ & $\begin{array}{c}\text { Enfoque } \\
\text { curricular }\end{array}$ & Estrategias didácticas \\
\hline Conductista & Cibernética & Tecnológico & $\begin{array}{l}\text { Estudiantes que reciben } \\
\text { información como } \\
\text { sinónimo de } \\
\text { conocimiento. }\end{array}$ \\
\hline Cognitivo & Mercadológica & Deliberativo & $\begin{array}{l}\text { Estudiantes que } \\
\text { construyen el } \\
\text { conocimiento a partir de } \\
\text { la experiencia, } \\
\text { aprendizaje } \\
\text { colaborativo y } \\
\text { deliberación }\end{array}$ \\
\hline Crítico & Contextual & Crítico & $\begin{array}{l}\text { Estudiantes que } \\
\text { construyen } \\
\text { conocimiento a partir } \\
\text { del diálogo entre el } \\
\text { conocimiento y la } \\
\text { realidad particular de } \\
\text { un contexto } \\
\text { determinado. }\end{array}$ \\
\hline
\end{tabular}

Fuente: Elaboración propia

Surgen una serie de preguntas, como las siguientes: ¿Qué características de ser humano están presentes en el Mandato Pedagógico Institucional y a qué tipo de persona nos refiere? La respuesta lleva a conocer la visión de mundo institucional. ¿Cuáles son las características del ser humano que se propone educar la carrera en cuestión? O dicho de otro modo ¿Con cuál visión de mundo coincide el perfil de ser humano que se pretende educar? La respuesta dirige a conocer la visión de mundo - ser humano que prevalece en esa propuesta curricular. ¿Coinciden, en su visión de mundo - ser humano, el Mandato Pedagógico Institucional y el perfil de egreso de la carrera? Finalmente, ¿contribuyen las estrategias didácticas al logro de la propuesta curricular y por ende al logro del Mandato Pedagógico Institucional? Si todas las respuestas coinciden en el enfoque pedagógico, es posible que se vaya en buen camino hacia el logro de la coherencia curricular.

\section{Conclusión}

La coherencia curricular es posible. Puede parecer abstracto e imposible de medir, no obstante, pueden darse algunos pasos para comenzar esta ruta, pocas veces andada, en el tema curricular.

Este trabajo aporta varios conceptos para caminar hacia el logro de la coherencia institucional. Tanto pedagogía -o ciencia que estudia el fenómeno educativo intencionado- como currículo -o estructura creativa para lograr un determinado perfil de ser humano- y didáctica -o instrumental creativo para lograr la propuesta curricular- orientan teóricamente a quienes ejecutan propuestas curriculares, tanto en el ámbito de la administración y ejecución como de la docencia, la investigación y la acción social.

La reflexión teórica tiene un sesgo importante, y es el hecho de motivar a salir del paradigma de los resultados para transitar hacia los procesos educativos, que deberían ser evaluados más por sus características y no tanto por sus resultados. Es decir, una propuesta educativa (su currículo y su didáctica) debería evaluarse 
observando los procesos que desarrolla promoviendo el aprendizaje y el logro de su perfil de egreso. La naturaleza humana no puede encajarse en una serie de resultados; hacerlo sería como tomar fotografías del fenómeno, sin tener la consciencia de que minutos después ya el fenómeno se modificó.

Las personas no son estáticas ni controlables. Por lo tanto, los procesos educativos, tanto a nivel curricular como didáctico, también son dinámicas humanas que no deberían pensarse, únicamente, desde una perspectiva de resultados. El asunto, entonces, se convierte en una interrogante, en términos de definir los criterios que orienten la observación de los procesos y que se constituyan en elementos de juicio para valorar si se logra o no el aprendizaje.

El punto expuesto en este trabajo, es que estos criterios, de manera consciente o inconsciente, corresponden a una determinada posición pedagógica o enfoque pedagógico o, también, asumir aportes de varios enfoques, desde una mirada ecléctica.

Toda institución educativa asume un compromiso con la formación de seres humanos, para que desarrollen determinadas características, lo que la aleja -o la acerca- a determinados enfoques pedagógicos. Lo interesante de la propuesta compartida en este trabajo, es que categoriza tres clases de miradas pedagógicas -o enfoques pedagógicos-.

¿Cuál es la prioridad en el aprendizaje según cada enfoque pedagógico propuesto en este trabajo? Para el enfoque conductista es memorizar la información para replicarla o utilizarla mediante ensayo y error en las distintas situaciones a que se enfrentan quienes aprenden, mientras que para el cognitivo la prioridad es construir la información mediante la experiencia de manera que se conviertan en parte de la estructura de la persona para que pueden ser adaptados a las distintas situaciones, y para el enfoque crítico, la prioridad es que el aprendizaje se haya logrado mediante diálogos entre la información y la realidad específica desde una perspectiva de derechos humanos -y que a la vez es dinámica- por lo que el aprendizaje se construye en determinados contextos en los cuales, quien aprende, asume una posición crítica. Puede decirse, en pocas palabras, que para el conductismo la memoria es clave, para el cognitivismo la experiencia es clave y para el enfoque crítico el diálogo, la interacción entre pares y la realidad es clave.

Por lo tanto, la tesis que se plantea es que cuando el enfoque pedagógico de la propuesta curricular y de las estrategias didácticas es el mismo, y cuando ambos factores responden al Mandato Pedagógico Institucional, entonces puede afirmarse que existe coherencia curricular. Aprender a reconocerlo es un proceso que, para que sea relevante, es colectivo, en manos de quienes ponen en práctica la propuesta curricular.

\section{ReFERENCIAS}

Aldana, C. (1996). Pedagogía general crítica (tomo II). Guatemala: Serviprensa Centroamericana.

Bolaños, C. (2015). Orientación para los procesos de diseño curricular. Costa Rica: Centro de Evaluación Académica, Universidad de Costa Rica. Recuperado de https://goo.gl/ijjjAH

Capra, F. (1992). El punto crucial. Argentina: Editorial Troquel.

Capra, F. (1991). El nuevo paradigma ecológico. Nueva Conciencia (22), 28-31. Recuperado de https:// goo.gl/3NSVym

Castells, M. (2002). La dimensión cultural de Internet. Institut de Cultura: Debates culturales. Recuperado de https:// goo.gl/zN4VfE

De Souza Silva, J. (2011). Hacia el 'Día Después del Desarrollo'. Descolonizar la comunicación y la educación para construir comunidades felices con modos de vida sostenibles. Asociación Latinoamericana de Educación Radiofónica - ALER. Recuperado de https://goo.gl/whMqex

Díaz, A. (2010). Didáctica y currículum. Convergencias en los programas de estudios. México: Editorial Paidós.

Escudero, J. (Ed). (1999). Diseño, desarrollo e innovación del currículum. Madrid, España: Síntesis Educación.

Freire, P. (1997) La Educación como práctica para la libertad. Madrid, España: Siglo XXI editores. 
Gimeno S., J. (2010). La función abierta de la obra y su contenido. Sinéctica. SciELO-Scientific Electronic Library Online. Recuperado de: https://goo.gl/bycwxA

Hawes, G. y Corvalán, O. (2005). Construcción de un Perfil Profesional. En Proyecto Mecesup Tal 0101, Documento de trabajo 1/2004. Talca, Chile: Instituto de Investigación y Desarrollo Educacional (IIDE). Recuperado de https://goo.gl/V2kcDS

González, V. (2017, enero). La Universidad de Costa Rica y su Mandato Pedagógico Institucional. En Memoria XV Encuentro Internacional por la Unidad de los Educadores, Pedagogía 2017. La Habana, Cuba.

González, V. (2014). Innovar en docencia universitaria: algunos enfoques pedagógicos. Intersedes - Revista Electrónica de las Sedes Regionales, Universidad de Costa Rica, 15(31), 50-68. Recuperado de https:// revistas.ucr.ac.cr/index.php/intersedes

Luzuriaga, L. (1962). La historia de la Educación y de la Pedagogía. Buenos Aires, Argentina: Losada.

Real Academia Española. (2014). Diccionario de la Real Academia Española. Recuperado de http://lema.rae.es/drae/

Ruiz, J. M. (2005), Teoría del Currículum: Diseño, Desarrollo e Innovación Curricular. Madrid, España: Editorial UNIVERSITAS, S.A.

Savater, F. (2016). Me falta la alegría. Pensadores del 21. Recuperado de https://goo.gl/E4P4Ls

Trilla, J., Cano, E., Carretero, M., Escofet, A., Fairstein, G., Fernández, J., y Vila, I. (2001). El legado pedagógico del siglo XX para la escuela del siglo XXI. Editorial GRAÓ. Barcelona, España.

Ysunza, M. (2010). Perfil de egreso y formación profesional: una estrategia metodológica en el diseño curricular. México: IISUE - Instituto de Investigaciones sobre la Universidad y la Educación de la Universidad Nacional Autónoma de México.

\section{Notas}

2 La M. Ev. Marta Picado Mesén, en el momento de la entrevista, ejerce el cargo de Directora del Centro de Evaluación Académica de la Universidad de Costa Rica.

3 La Dra. Eleonora Badilla Saxe, en el momento de la entrevista, ejerce el cargo de Directora de la Red Institucional de Evaluación Docente de la Vicerrectoría de Docencia de la Universidad de Costa Rica.

4 La Dra. Flora Salas Madriz, en el momento de la entrevista, ejerce el cargo de Directora de Políticas, Programas y Proyectos de la Vicerrectoría de Docencia de la Universidad de Costa Rica.

5 La Dra. Patricia Marín Sánchez, en el momento de la entrevista, ejerce el cargo de Directora del Departamento de Docencia Universitaria de la Escuela de Formación Docencia, Facultad de Educación, Universidad de Costa Rica.

6 La Dra. Susan Francis Salazar, en el momento de la entrevista, ejerce el cargo de Directora de la Unidad de Apoyo a la Docencia Mediada con Tecnologías de la Información y Comunicación (METICS) y coordina el proyecto Multiversa, ambos de la Vicerrectoría de Docencia, Universidad de Costa Rica.

\section{BY-NC-ND}

